\title{
A man with an enlarging foot mass
}

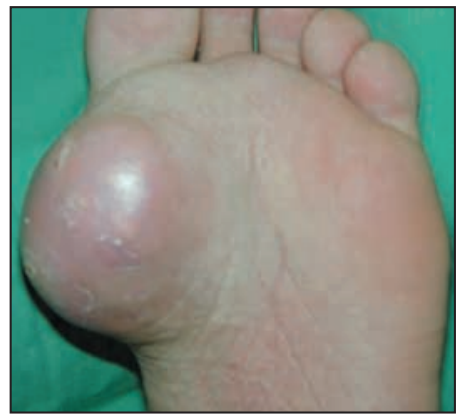

A 32-year-old man was referred to our orthopedic service for evaluation of a left plantarmedial forefoot mass that had been present for at least 2 years. He had had intermittent foot pain in the past but currently denied any pain or difficulty walking, even with footwear. He had received dialysis for the past 6 years for end-stage renal disease of unknown cause. He had no history of diabetes, gout, pseudogout or injury or surgery to the foot or ankle, but he did have cerebral damage due to solvent and alcohol abuse.

The large mass at the plantarmedial aspect of the distal first metatarsal region (Fig. 1) was deep to the skin, mobile and nontender. There was no erythema, open wound or drainage. Radiography showed a large, densely calcified multilobular mass extending from the proximal end of the first metatarsal distally to the hallux interphalangeal joint, with no bony erosions, consistent with tumoral calcinosis (Fig. 2). Routine laboratory tests, including serum calcium levels $(2.5 \mathrm{mmol} / \mathrm{L})$, were normal, but his serum phosphate level was elevated at 3.1 (normally 0.8-1.6) mmol/L.

The patient was asymptomatic, and thus no treatment was initiated. However, 1 year later, $\rightarrow$ the mass was larger and was causing pain with walking. The patient had a painful restriction of the range of motion of his first metatarsophalangeal joint. After an MRI confirmed the large mass, the man opted for surgical resection, which re-

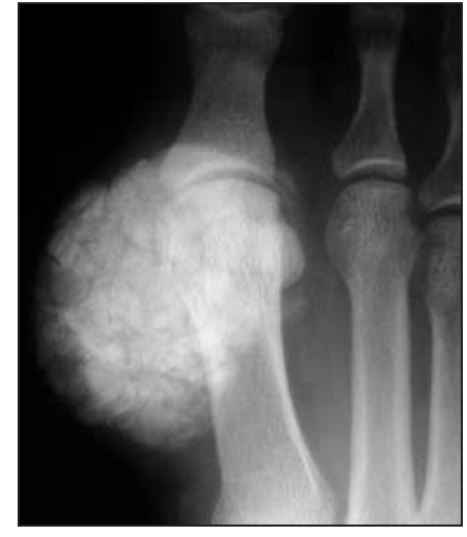

vealed a firm, multilobular, nonencapsulated tumour $(6 \times 5 \times$ $4 \mathrm{~cm}$ ) (Fig. 3) that exuded creamy, yellow-white, thick pasty discharge and was entirely extraosseous and extra-articular. Cultures were negative for bacteria, fungi and tuberculosis. Microscopic examination showed granular calcified material bordered by a chronic inflammatory cell infiltrate including mononuclear and multinucleated macrophages, osteoclast-like giant cells and fibroblasts, consistent with tumoral calcinosis. ${ }^{1,2}$ The patient had a good postoperative recovery, with no tumour recurrence or pain 6 months after surgery.

Tumoral calcinosis is characterized by the periarticular deposition of calcium phosphate primarily in the upper and lower extremities, ${ }^{2}$ usually in the setting of normocalcemia and hyperphosphatemia, ${ }^{2,3}$ but occasionally with normal phosphate ${ }^{4}$ or elevated 1,25-dihydroxyvitamin D levels. ${ }^{2}$ The extraskeletal softtissue masses occur because of excess calcium phosphate, which surpasses solubility limits ${ }^{1}$ and results in hydroxyapatite crystals and noncrystalline calcific deposits. ${ }^{2}$ Possible causes include renal failure, ${ }^{1,4}$ hyperparathyroidism, ${ }^{1}$ trauma, ${ }^{1,2,4}$ familial predisposition $^{2}$ and idiopathic causes. ${ }^{4}$ The pathogenesis may involve increased renal reabsorption of phosphate at the proximal tubule. ${ }^{3}$ Lesions may be massive,

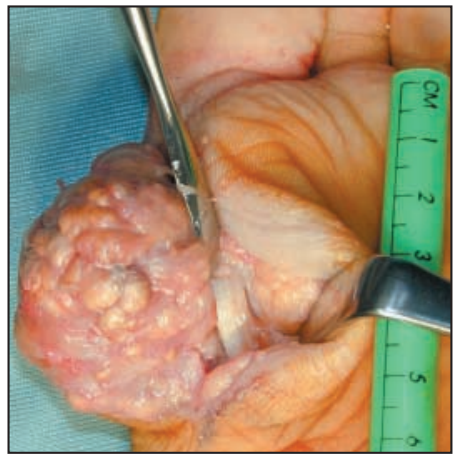

resulting in disfigurement and functional impairment. ${ }^{2,45}$

The differential diagnosis includes other benign or malignant tumours, ectopic ossification, metastatic calcification, gout, pseudogout, calcific bursitis, tendinitis, vitamin $\mathrm{D}$ toxicity or deformities caused by neuropathic arthropathy. ${ }^{1}$ Tumoral calcinosis has a characteristic radiographic appearance and histology, and it is also distinguished by elevated serum phosphate levels in some cases. Treatment options include dietary phosphorus deprivation and phosphate-binding antacids, ${ }^{1}$ parathyroidectomy ${ }^{1}$ and surgical excision. ${ }^{1,45}$ Recurrence after surgery occurs in about $60 \%$ of cases. $^{5}$

\section{Ali Zahrai \\ Deloar Hossain \\ John M. Embil \\ Elly Trepman \\ University of Manitoba \\ Winnipeg, Man.}

\section{References}

1. Tezelman S, Siperstein AE, Duh QY, Clark OH. Tumoral calcinosis: controversies in the etiology and alternatives in the treatment. Arch Surg 1993;128:737-45

2. Slavin RE, Wen J, Kumar D, Evans EB. Familial tumoral calcinosis: a clinical, histopathologic, and ultrastructural study with an analysis of its calcifying process and pathogenesis. Am 7 Surg Pathol 1993;17:788-802.

3. Mitnick PD, Goldfarb S, Slatopolsky E, Lemann J, Gray RW, Agus ZS. Calcium and phosphate metabolism in tumoral calcinosis. Ann Intern Med 1980;92:482-7.

4. Knowles SAS, Declerck G, Anthony PP. Tumoral calcinosis. Br 7 Surg 1983;70:105-7.

5. Smack DP, Norton SA, Fitzpatrick JE. Proposal for a pathogenesis-based classification of tumoral calcinosis. Int 7 Dermatol 1996;35:265-71. 